\title{
A Study of WI-FI based System for Moving Object Detection through the Wall
}

\author{
Gaurav K. Nanani \\ PG Research Scholar \\ Dept. of Electronics \& Communication \\ RK University, Rajkot, India
}

\author{
Kantipudi M V V Prasad \\ Assistant Professor \\ Dept. of Electronics \& Communication, \\ RK University, Rajkot, India
}

\begin{abstract}
Mostly WI-FI signals are used as information carrier signals between two stations one is transmitter and another is receiver. This paper gives review about those systems (Ultra Wideband System, WI-VI, WI-SEE) which are operates on WI-FI signal, in which WI-FI signals are not just the information carrier signal, but in these systems WI-FI signals are used to see moving object through wall. This review is initial part of the research for developing the algorithm to extend the results, which are achieved from the previous systems.
\end{abstract}

\section{Keywords}

Flash effect, WISEE, WIVI, Doppler shift, MIMO

\section{INTRODUCTION}

The use of WI-FI signal is not only as an information carrier; these WIFI signals can be used to track the moving object behind the wall or can say in a close room. This idea can be used to minimize the causalities in standoff and hostage condition, emergency responder can be used to see through wall, rubber or collapsed structure.

To recognize moving object through wall the WIFI signal transmitted in the direction of wall, this results in two problem (I) Flash effect, (II) magnitude of signal reduced by three to five times after traversing the wall. To solve these problems systems are developed and experiments performed on those systems to achieve the desired results. The radar community developed a system named Ultra-Wideband. This system detects human behind the wall and shows moving blobs in output screen (watch the video at [3]). To overcome the drawbacks of this system two new system were developed named WI-VI and WISEE. These both systems use WI-FI signal to recognize the moving object behind the wall. WISEE system works on the Doppler Sift principle, for the human gesture it gives the different Doppler pattern according to the movement of any human . But the problem with this system is, very small change in Doppler shift for the human gesture which is very difficult to detect. On the other hand WI-VI system also use WI-FI signals to recognize the moving object behind the wall, this system use the nulling technique to remove the flash effect. This system not require large antenna array, it only need three small antenna MIMO radio interface.

Flash effect term refers to "The reflection from the entire stationary object behind the wall rather than just wall", which is much stronger than the reflection from the object inside the closed room.

\section{ULTRA WIDE-BAND SYSTEMS}

This system is recently developed by the radar community to detect the moving object behind the wall which give moving blobs as a result for the moving object behind the wall [5]. This system separates reflection from wall or other static object and the reflection from moving object behind the wall based on their arrival time, so it must required to identify delays of even sub-nanoseconds.

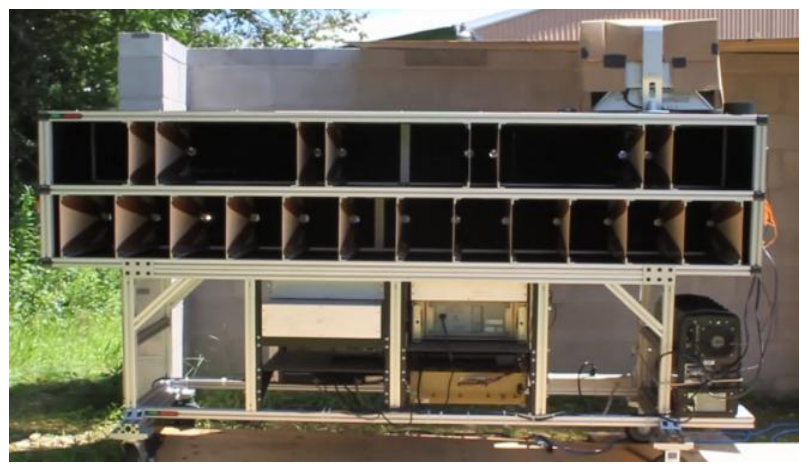

Fig.1: Ultra Wide-Band System [3]

The system shown above in Fig.1 requires $2 \mathrm{GHz}$ of bandwidth, a very large power source, and around 8-foot long antenna array [6] and most important parameter is that the large power in such a wide spectrum is infeasible for entities other than the military application.

\section{WISEE}

WISEE is a gesture recognition system that utilizes wireless signals to recognition of human gesture. This system can recognize the human gesture without requiring any sensing device on the human body. The required prototype for WISEE system is developed using USRP-N210.

WISEE system use the property of Doppler shift [8], Doppler shift is the frequency change of transmitted wave as its source moves relative to the observer. There will be multipath reflection from the human body, and then human gestures results in pattern of Doppler shift at the system receiver. So, the movement of user away from the receiver results in negative Doppler shift, and movement of user towards the receiver results in positive Doppler shift. The challenge for this system was that result of the human gesture gives very small change in Doppler shifts that can be very hard to detect from WI-FI transmission. Typically movement around 0.5 $\mathrm{m} / \mathrm{sec}$ results in $17 \mathrm{~Hz}$ Doppler shift for the $5 \mathrm{GHz}$ WI-FI transmission. For the gesture recognition it is required to detect the Doppler shifts of few Hertz from $20 \mathrm{MHz}$ WI-FI signals. This solution of this problem is achieved by transforming the signal which are received from moving object, in to narrowband pulse with a bandwidth of few Hertz, then system tracks the frequency of this narrowband pulses to 
detect the small Doppler shift. Let an object moving at speed of $v$ with angel of $\theta$ from the receiver results in Doppler shift given by the following equation [2] where $C$ is speed of light and $f$ is the center frequency of the system transmitter.

$$
\Delta f \propto \frac{2 v \cos (\theta)}{C} f
$$

In home there may have more than one person who can affects the wireless signals. This problem is solved by MIMO capability which is inherent to $802.11 \mathrm{n}$, to focus the gesture from a particular human. The wireless reflection from all the humans can be separated using MIMO receiver.

Finally The experiments were performed for line-of-site, non-line-of-site, and through wall where the person is in different room from the wireless transmitter and receiver and achieved results are as follow:

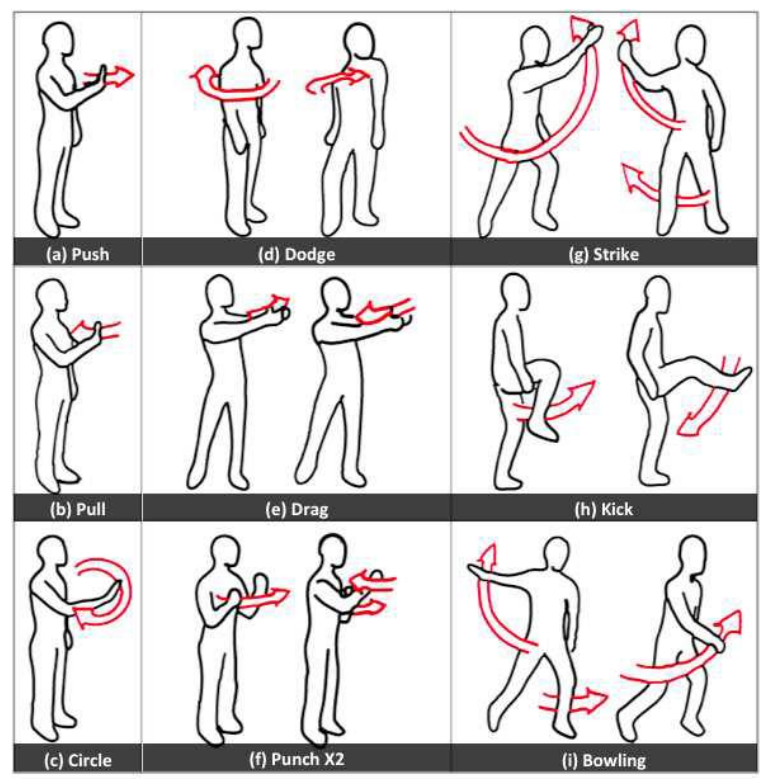

Fig.2: Gesture Sketches [2]

- WISEE system can track the 9 human body gesture shown in Fig.2, with 94\% accuracy.

- Using four receiving antenna and one transmitting antenna WISEE can achieve $60 \%$ accuracy.

- Using five receiving antenna and single transmitting antenna WISEE can perform the human gesture classification in presence of other three people who are performing random gesture.

\section{WIVI}

To address the limitation of the ultra wide-band system, in 2012 an attempt was made to use WI-FI signal to see through wall. The objective of this attempt was to enable the technology of see through wall at low-bandwidth, low-power, compact size, and accessible to non-military entities. With these objectives WI-VI system was developed that employs WI-FI signals in the $2.4 \mathrm{GHz}$ ISM band. This system limits its operating frequency to a $20 \mathrm{MHz}$ wide WI-FI channel, instead of wide spectrum in Ultra Wide-Band system to remove the flash effect. This system also eliminates the use of large antenna array by using only 3 antenna MIMO radio instead of 8 -foot long antenna array.

This system removes the flash effect using nulling (received signals sums up to zero) technique at the receiving antenna. WI-VI system has two transmission antenna and single receiving antenna. This WI-FI system operates in two stages, in first stage system estimate the channel from both its transmitter antenna to the receiver antenna. In second stage use the channel estimation from stage one to null the signal at the receiving antenna.

To track the moving object WI-VI use technique named inverse synthetic aperture radar (ISAR), as shown in Fig.3 an antenna array is able to track the moving object by spatially spaced antennas and process this information to achieve the direction of that moving object with respect to antenna array. On the other side ISAR use only single receiver antenna to track the moving object, here in ISAR moving object or human works as an antenna array. Here at any point in time, we capture a single measurement.
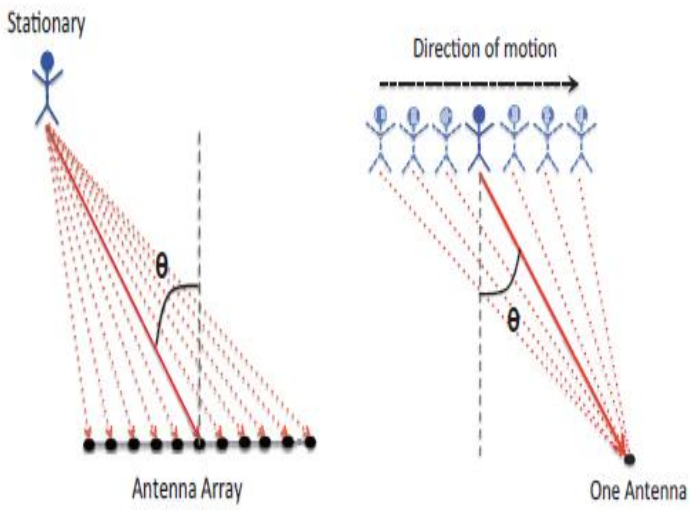

Fig.3: antenna array and ISAR [1]

\section{TRACKING A SINGLE HUMAN}

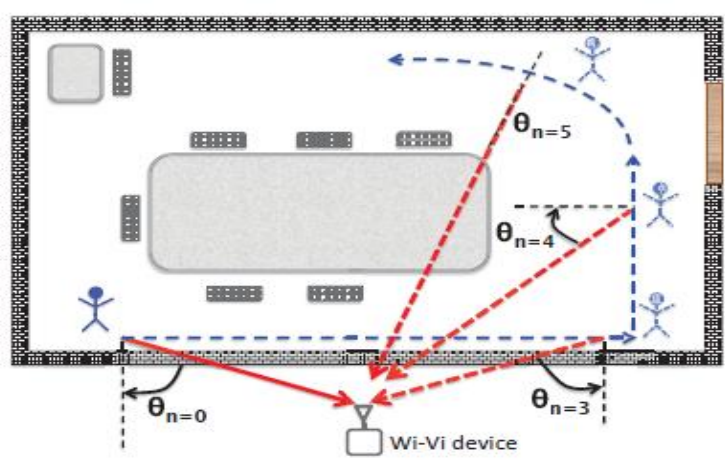

Fig.4[a]: Experimental setup [1] 


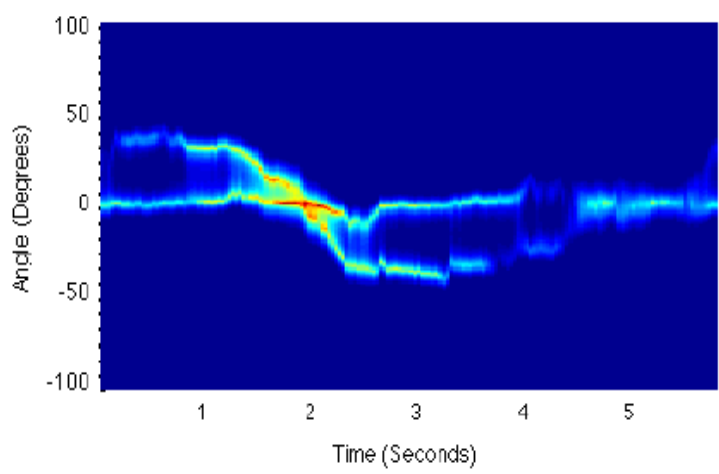

Fig.4[b]: WI-VI output [1]

Fig.4 shows one of the experiments performed on WI-VI system. Fig.4 [a] is experimental setup and Fig.4 [b] is the magnitude response of the reflected signal from the moving object. There is one curved line in Fig.4 [b] which shows tracking of human motion. Spatial angle $\Theta$ is the angle between the line connecting the human to WI-VI and normal to motion. Observe that the $\Theta$ is positive when vector from human to WI-VI and vector of the motion are in same direction and $\Theta$ is negative when these both vectors are in opposite direction.

The related prototype for WI-VI system is developed using USRP N210 radios and algorithm [7] is performed on MATLAB R2012a under Ubuntu and experiments were performed in two offices. Achieved results from the experiments are as follow:

- This device can track the human up to range of 8 meters between transmitter and object with $75 \%$ accuracy and can't track the human at distance of 9 meters.

- WI-VI can track the moving object up to the 8" thicker concrete wall, 6" thicker hollow wall and 1.75 " solid wooden doors.

- As WI-VI replacing the antenna array by ISAR means that the angular resolution in this system depends on amount of movement. It removes clutter from all static object rather than just wall.

- From the Fig.4 [b] it can be concluded, at output we can achieve only magnitude plot according to the movement of object it doesn't provide the shape of that object.

\section{COMPARATIVE ANALYSIS}

Here we have discussed three systems. The various parameters of these systems are compared here. From This comparison it can be concluded that the WI-VI system is much better in terms of all parameters which are compared here. The more work on the WI-VI system can give us more advantages and system can work more accurately.

Table 1. Comparative Analysis table

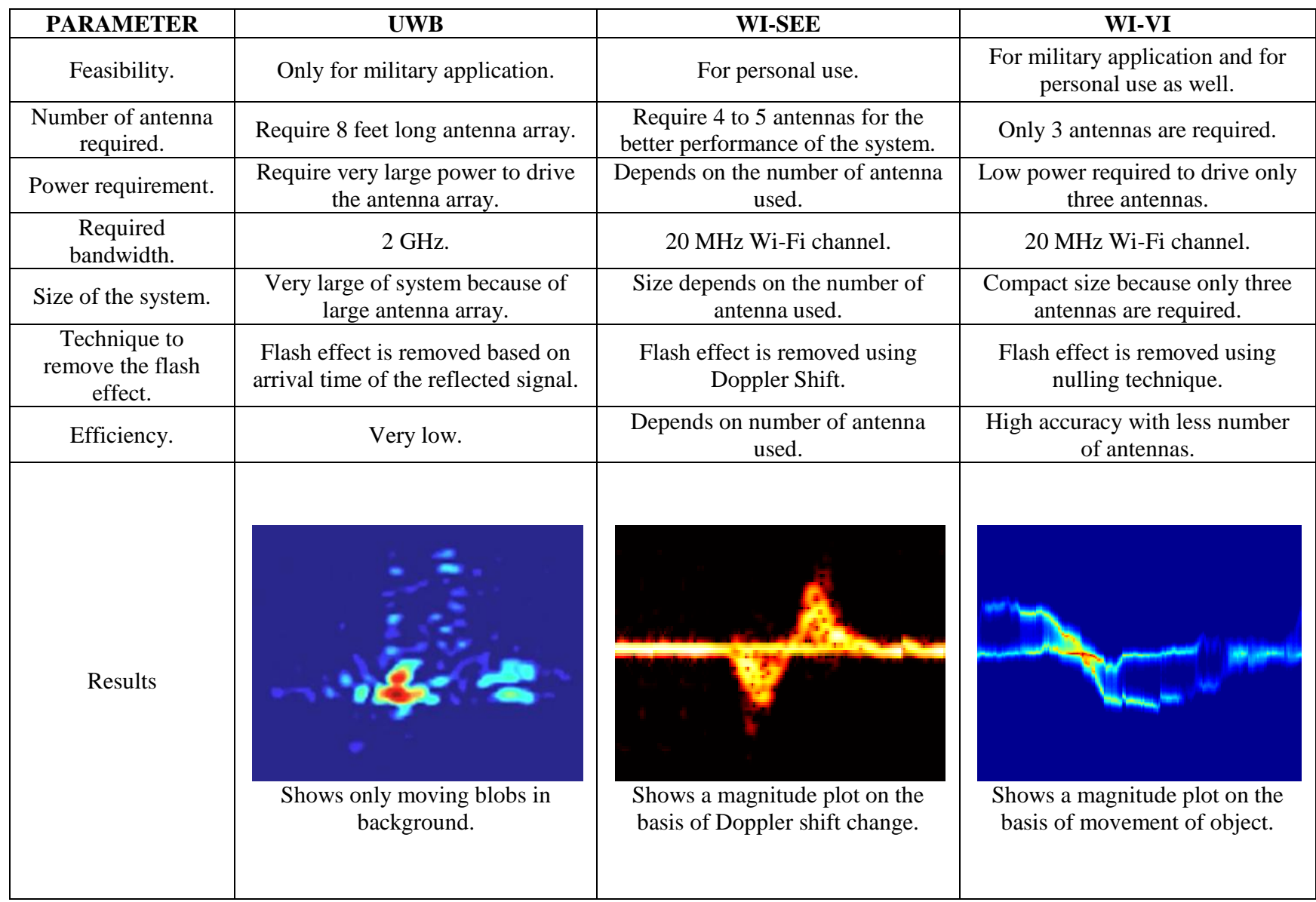




\section{CONCLUSION}

After detail analysis of these three system it can be concluded that the need of human recognition through wall is the most required in the field of human safety in the hostage situation, and have good scope to extend achieved results with the WI-VI system. New efficient algorithms can be developed to identify the shape of the object rather than just magnitude plot of the moving object. Using the different algorithms some parameters can be extended and system can be made of high performance.

\section{ACKNOWLEDGMENT}

I am very much thankful to Mr. $K M V V$ Prasad for success full completion of this review paper. I also acknowledge my friends for their kind support.

\section{REFERENCES}

[1] F. Adib and D. Katabi, "See through wall with WI-FI", Massachusetts Institute of Technology.

[2] Q. Pu, S. Gupta, S. Gollakota, and S. Patel, "Wholehome gesture recognition using wireless signals", University of Washington.

[3] "Seeing through walls" - MIT's Lincoln Laboratory, http://www.youtube.com/watch?v=H5xmo7iJ7KA.
[4] G. Charvat, L. Kempel, E. Rothwell, C. Coleman, and E. Mokole, "through-dielectric radar imaging system" IEEE Trans, Antennas and Propagation, 2010.

[5] Y. Yang and A. Fathy, "Design and implementation of a low-cost real-time ultra-wide band see-through-wall imaging radar system", IEEE/MTT-S International Microwave Symposium, 2007.

[6] G. Charvat, L. Kempel, E. Rothwell, C. Coleman, and E. Mokole, "An ultrawideband (UWB) switched-antennaarray radar imaging system”, IEEE ARRAY, 2010.

[7] F. Soldovieri and R. Solimene, "Through-wall imaging via a linear inverse scattering algorithm", IEEE Geoscience and Remote Sensing Letters, 2007.

[8] S. Gupta, D. Morris, S. Patel, and D. Tan, "Soundwave: using the doppler effect to sense gestures", HCI 2012.

[9] W. Carrara, R. Goodman, and R. Majewski, "Spotlight Synthetic Aperture Radar: Signal Processing Algorithms", Artech House, 1995. 